\title{
Reframing noncommunicable diseases and injuries for the poorest Malawians: the Malawi National NCDI Poverty Commission
}

\author{
Katie Cundale ${ }^{1}$, Emily Wroe ${ }^{1}$, Beatrice L. Matanje-Mwagomba ${ }^{2,3}$, Adamson S. Muula ${ }^{3}$, Neil Gupta ${ }^{4}$, \\ Josh Berman ${ }^{5}$, Noel Kasomekera ${ }^{1,2}$, Jones Masiye ${ }^{2}$
}

1. Partners in Health/Abwenzi Pa Za Umoyo, Neno, Malawi

2. Noncommunicable Diseases and Mental Health Control Unit, Directorate of Clinical Services, Ministry of Health, Lilongwe, Malawi

3. Department of Public Health, College of Medicine, University of Malawi, Blantyre, Malawi

4. NCD Synergies, Boston, Massachusetts, USA

5. Dignitas International, Zomba, Malawi

Correspondence: Dr Emily Wroe (ewroe@pih.org)

\begin{abstract}
Noncommunicable diseases and injuries (NCDIs) account for nearly $70 \%$ of deaths worldwide, with an estimated $75 \%$ of these deaths occurring in low- and middle-income countries. Globally, the burden of disease from noncommunicable diseases (NCDs) is most often caused by the "big 4," namely: diabetes, cardiovascular diseases, cancer, and chronic lung diseases. However, in Malawi, these 4 conditions account for only $29 \%$ of the NCDI disease burden.

The Malawi National NCDI Poverty Commission was launched in November 2016 and will describe and evaluate the current NCDI situation in Malawi, with a focus on the poorest populations. The National Commission will investigate which NCDIs cause the biggest burden, which are more present in the young, and which interventions are available to avert death and disability from NCDIs in Malawi, particularly among the poorest segments of the population.

The evidence gained through the work of this Commission will help inform research, policy, and programme interventions, all through an advocacy lens, as we strive to address the impact of NCDIs among all populations in Malawi.
\end{abstract}

\section{Why a Commission on NCDIs?}

Noncommunicable diseases and injuries (NCDIs) account for nearly $70 \%$ of deaths worldwide, with an estimated $75 \%$ of these deaths occurring in low- and middle-income countries. ${ }^{1}$ NCDIs are usually associated with the rich, the elderly, or urban populations. ${ }^{2}$ Furthermore, the interventions aimed around NCDIs often follow the behavioural change model, based on 4 common risk factors: unhealthy diets, harmful use of alcohol, smoking, and physical inactivity. ${ }^{1}$ Yet while global and national policies tend to focus on these populations and this model, is this reflecting the lived realities of all populations? For those that suffer from NCDIs not related to behavioural risk factors, is this focus missing significant disease burdens? In 2013, Malawi participated in an international forum discussing precisely these questions and signed on to commit to address NCDIs in impoverished settings, with a focus on the young, described by Binagwaho et al. in The Lancet in 2014. ${ }^{3}$

The Malawi National NCDI Poverty Commission, 1 of 11 National NCDI Poverty Commissions, was launched in November 2016 and will describe and evaluate the current NCDI situation in Malawi, with a focus on the poorest populations. The National Commission is investigating which NCDIs cause the biggest burden, which are more prevalent in the young, and which interventions are available to avert death and disability from NCDIs in Malawi, particularly among the poorest segments of the population. It is anticipated that the results from these analyses will help guide Malawian policy and set priorities to inform efforts to reduce the NCDI burden in a comprehensive way across all sectors of the population.
Malawi's National NCDI Poverty Commission is contributing data and expertise to the Global Commission: the Lancet Commission on Reframing NCDIs for the Poorest Billion (see: www.ncdipoverty.org). This Global Lancet Commission is working with 11 different countries that encompass the majority of the world's poorest, with the goal to elucidate the global burden of NCDIs among the world's poorest billion and to describe and evaluate the current interventions aimed at addressing them. In doing so, the Commissions will refocus our understanding of the NCDI burden to ensure it encompasses all those suffering from NCDIs.

\section{NCDIs in Malawi}

In Malawi, according to Global Burden of Disease (GBD) study estimates, $38 \%$ of the burden of disease from noncommunicable diseaes (NCDs) is caused by the "big 4," namely: diabetes, cardiovascular diseases (CVD), cancer, and chronic lung diseases. ${ }^{4}$ This is in stark contrast to highincome countries, where these same 4 conditions comprise $48 \%$ of the disease burden (Figure 1). ${ }^{4}$ These 4 conditions are generally thought to be related to modifiable and shared risk factors: tobacco use, unhealthy diets, physical inactivity, and the harmful use of alcohol. ${ }^{1}$ In fact, only $17 \%$ of the NCD disability-adjusted life years (DALYs) in Malawi are from the big 4 categories with any behavioural or metabolic attribution, compared to $30 \%$ for high-income countries. ${ }^{4}$ Furthermore, the majority of the NCD burden- $62 \%$-is caused by other conditions outside of these 4 classic NCDs, ${ }^{4}$ which do not necessarily share the same risk factors. This includes a large set of conditions, such as rheumatic heart disease, mental illness, road traffic injuries, and type 1 diabetes. In Malawi, our analysis with GBD data also shows that the burden of NCDIs falls disproportionally on the young, with $82 \%$ of 
the injury burden and $60 \%$ of the NCD burden occurring in those less than 40 years of age, compared to $40 \%$ and $18 \%$ in high-income countries, respectively. ${ }^{4}$ This is consistent with data from sub-Saharan Africa generally, where the probability of dying from or living with a disability related to an NCDI is highest for persons aged between 30 and 70 years. ${ }^{5}$ These NCDI conditions outside of the big 4those that have different risk factors and disproportionately affect the young-are an important and significant piece of the NCDI burden in Malawi, presenting a critical and complementary set of conditions to address in policy and interventions.

\section{Current interventions in Malawi}

Much of what is currently known in Malawi about NCDIs, as well as the interventions targeting them, centres around hypertension and diabetes. According to the 2009 STEPS Survey, it was estimated that $33 \%$ of Malawian adults had high blood pressure and $6 \%$ had diabetes. ${ }^{6}$ These results, coupled with the global call for action against NCDs, led to the national response: the government of Malawi, through the Ministry of Health, included NCDs in the Health Sector Strategic Plan. ${ }^{7}$ This was followed by the establishment of a National Coordinating Unit-the NCDs and Mental Health Unit. In collaboration with partners and stakeholders, the Unit has since developed plans and interventions to address NCDIs.

The first National Action Plan for the Prevention and Management of NCDs (2012-2016) was launched in December 2013 during the World Diabetes Day commemoration. The Unit has further initiated a number of projects to improve prevention and enhance care for some NCDs. The first was the adaptation and launch of the Package of Essential NCD (PEN) interventions ${ }^{8}$ by the World Health Organization (WHO) in 2012, with pilot implementation at Kasungu District Hospital. The WHO PEN interventions, which include improving primary care capacity for early diagnosis, appropriate management for chronic NCDs, as well as health promotion, are currently being scaled up through a World Diabetes Foundation supported project that was launched in September 2015 to improve access, care, and prevention of hypertension and diabetes. ${ }^{9}$

Other interventions include: a Sports for Health initiative that aims to raise awareness about prevention and control of NCDs through physical activities; the introduction of a Human Papillomavirus (HPV) vaccine for adolescent girls for primary cervical cancer prevention in Zomba and Rumphi Districts; the development of the National Alcohol Policy, which aims to reduce the harmful use of alcohol; and the further implementation of One Stop Centres for genderbased violence, including sexual, physical, and emotional abuse. Further programmess are being implemented by nongovernmental organisations across the country, with many focusing on the integration of HIV and NCD care.

These represent encouraging steps towards reducing deaths and disabilities related to NCDIs, and the National Commission posits that complementary approaches are needed in order to tackle the broader burden of NCDIs, particularly among Malawi's poorest. For those living in absolute or extreme poverty, the risk factors for NCDs are much more likely to relate to genetics, infections, and harmful environments, ${ }^{2}$ rather than behavioural risk factors, such as diet and exercise. This communication, therefore, focuses on the question of whether the current understanding of NCDIs in Malawi is truly reflecting the reality of the poorest, and whether the subsequent policies and interventions currently in place are helpful to those who suffer from NCDIs unrelated to lifestyle factors. Is there more that should be considered, beyond what is already being addressed?

\section{Overview of the Lancet NCDI Poverty Commission}

NCDIs afflicting the poorest billion do not individually reflect a major cause of death or disability worldwide, but put together, "they could account for more than a third of the disease burden among those living in extreme poverty, with more than two-thirds of this burden concentrated among individuals younger than 40 years." 2 The types of NCDIs affecting these populations could include conditions, such as type 1 diabetes, rheumatic heart disease, cervical cancer, epilepsy, mental health disorders, and injuries from multiple sources, including domestic violence and road traffic injuries. ${ }^{2}$ With the acknowledgment that there may be an unnoticed burden affecting the poor, the Global Lancet Commission was launched to directly describe this problem among the "poorest billion".

The Lancet Commission defines the poorest billion, or those classified as being in "severe poverty", as anyone deprived in $50 \%$ or more of the 10 indicators in the Multidimensional Poverty Index (MPI) (Table 1). ${ }^{10}$

Table 1: Multidimensional poverty index

\begin{tabular}{c|l}
\hline Dimensions of poverty & Indicators \\
\hline Education & Years of schooling \\
& Child school attendance \\
\hline Health & Child mortality \\
& Nutrition \\
\hline \multirow{2}{*}{ Living standards } & Electricity \\
& Improved sanitation \\
& Improved drinking water \\
& Flooring \\
& Cooking fuel \\
& Assets ownership \\
\hline
\end{tabular}

\section{Overview of the Malawi National NCDI Poverty Commission}

Led by the Ministry of Health, the Malawi National NCDI Poverty Commission consists of country representatives working with the Global Commission, with membership from the NCD Unit, Kamuzu Central Hospital, Queen Elizabeth Central Hospital, Mzuzu Central Hospital, the University of Malawi College of Medicine, Partners In Health, Dignitas International, Malawi Epidemiology and Intervention Research Unit (MEIRU), Lighthouse Trust, Partners in Hope, and Baobab Health Trust. The team will analyse epidemiologic and other nationally available data, explore current interventions, leverage expert opinions in Malawi, and describe the lived reality of NCDIs through patient and provider perspectives. The analysis will start with an in-depth look at the Global Burden of Disease data 


\section{Percent of DALYs in Broad NCD Categories}
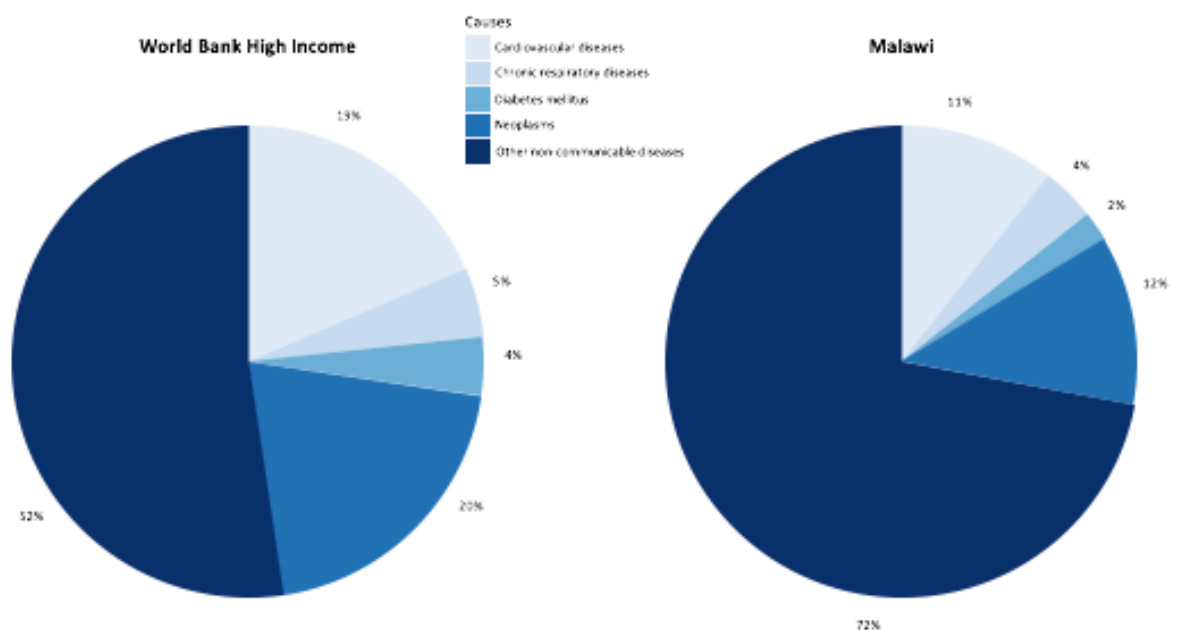

Figure 1: The contributing disease categories for noncommunicable disease (NCD) disabilityadjusted life years (DALYs) in Malawi, compared to high-income countries ${ }^{3}$

Using 2013-2014 Multiple Indicator Cluster Survey (MICS) data to determine MPI, $56 \%$ of Malawi's population is considered multidimensionally poor. $^{10}$ On average, the population is deprived in just over $47 \%$ of weighted indicators. However, looking at the MPI more closely reveals that $64.8 \%$ of Malawi's population is considered deprived in $50 \%$ or more of the indicators-the Lancet Commission's definition of the "poorest billion". Further breakdown of this figure shows that those in severe poverty vary geographically (Figure 2), with $71.6 \%$ of the population in rural areas and only $22.6 \%$ of urban

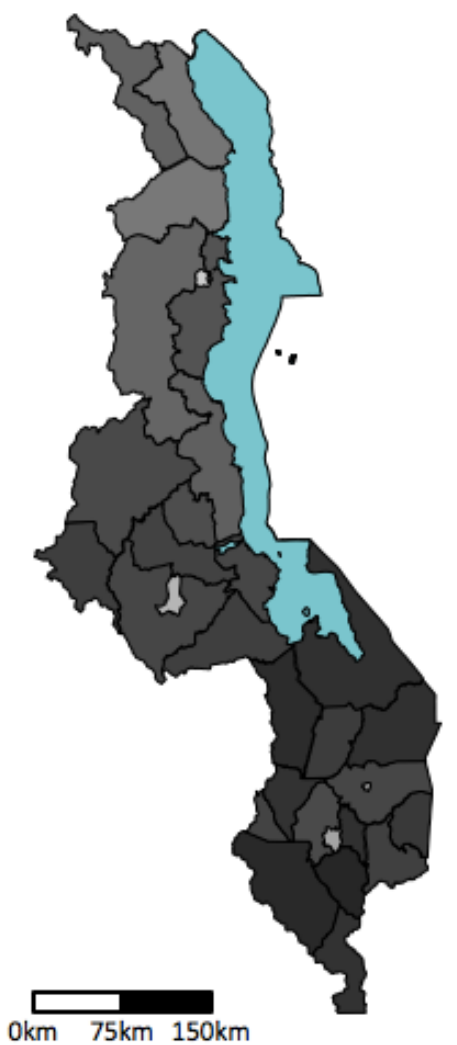

\section{Percent in Poorest Billion}

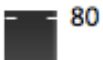

60

$-40$

20

Figure 2: The geographic distribution of Malawi's population considered to be living in severe poverty ${ }^{10}$

from the Institute for Health Metrics and Evaluation-a set of tools that draws on an extensive set of data available from over 2000 researchers in more than 130 countriesallowing comparison of prevalence as well as harm for more than 300 diseases and injuries by age and sex, and over time since $1990 .{ }^{11}$ Complementing this analysis, the Commission will leverage local researchers and datasets, as well as a comprehensive literature review. This will position the Malawi National Commission to support the analysis of the Global Commission on the NCDI burden among the poorest, ensuring that the burden of disease in Malawi is accurately described and national priorities set based on available evidence. populations being deprived in $50 \%$ or more of indicators. ${ }^{10} \mathrm{It}$ is this population that the National Commission will address for the burden of and interventions for NCDIs - the almost $65 \%$ of the Malawian population living in extreme poverty.

\section{Goals of the Malawi National NCDI Poverty Commission}

The overall goals of national-level Commissions are:

1. Analyse the burden of NCDIs among the world's poorest billion people in terms of fatal and nonfatal outcomes, as well as risk factor attribution;

2. Determine priority strategies to address this burden in specific countries; and

3. Identify the gap in resources that will need to be addressed leading up to 2030 in order to implement those strategies in terms of finances, human resources, and governance. ${ }^{12}$

Within each national Commission are 4 working groups, each tasked with a specific aim that will contribute to these overall goals: (1) establish the baseline NCDI epidemiologic and socioeconomic situation; (2) describe the current state of NCDI interventions and set priorities for NCDI interventions; (3) describe the current financing for NCDIs and quantify resource needs for priorities; and (4) describe past and current governance of NCDIs and highlight the patient experience of those affected by NCDIs. ${ }^{12}$

By participating in this Commission, Malawi will be able to not only better understand the NCDI burden within the nation, but will also be able to learn from and help others identify the most effective interventions to address NCDI burden among the poorest. It is a knowledge building and sharing platform, and the team is currently participating in World Bank Knowledge Exchanges and contributing to biweekly newsletters shared among the 10 other national Commissions.

By determining the socioeconomic and geographic burden of NCDIs within Malawi, the Commission will be able to inform government and donor priorities based on epidemiologic, financial, and governance evidence. 
Ultimately, the results from the analyses performed at the global and national scales will help inform NCDI policy development and refinement. As the Malawian National Action Plan on NCDs ended in 2016, it is hoped that the outputs from this Commission will be able to contribute to the new action plan. Additionally, given the inclusion of NCDs in the Essential Health Package (EHP) in Malawi's Health Sector Strategic Plan, the Commission's findings around the broader set of important NCDI conditions, particularly among the young, can help guide important steps and interventions toward realisation of the EHP. By integrating with national policies and strategies in the NCD space, the Commission will pursue mechanisms to integrate the findings into ongoing interventions and monitoring and evaluation processes, in order to track progress for NCDIs in Malawi's impoverished population over time. The Commission aims to disseminate a complete report on NCDIs of the poor in Malawi-encompassing burden, interventions and their cost, and economic landscape-by September 2017.

The focus of this Commission is on the poorest, with a goal to build upon the current efforts to address NCDIs in Malawi and to help reframe the global and national agenda around noncommunicable diseases to ensure that it includes the comprehensive burden of disease, as well as the poorest of the poor. The process undertaken by the Malawi National NCDI Poverty Commission is an evidence-driven effort to inform research, policy, and programme interventions-one that is rooted in advocacy as we collectively strive to address the impact of NCDIs in Malawi.

\section{Acknowledgements}

This article was written on behalf of the members of the Malawi National NCDI Poverty Commission:

Moffat Nyirenda, Lilian Chunda, Ronald Manjomo, Elizabeth Dunbar, Sam Phiri, Chawanangwa Lupafya, Dan Namarika, John Chipolombwe, Michael Udedi, Mia Crampin, Satish Gopal, Leo Masamba, Dominic Nkhoma, Kelias Msyamboza, Colin Pfaff, Luckson Dullie, Grace Banda, and Sosten Chilumpha

\section{Competing interests}

All authors declare that they have no competing interests related to this work.

\section{References}

1. World Health Organization (WHO). Noncommunicable diseases. 2015. Available online from: http://www.who.int/mediacentre/ factsheets/fs355/en/. Accessed 15 Jan 2017.

2. Bukhman G, Mocumbi AO, Horton R. Reframing NCDs and injuries for the poorest billion: a Lancet Commission. Lancet. 2015 Sep 26;386(10000):1221-2.

3. Binagwaho A, Muhimpundu MA, Bukhman G; NCD Synergies Group. 80 under 40 by 2020: an equity agenda for NCDs and injuries. Lancet. 2014 Jan 4;383(9911):3-4.

4. Institute for Health Metrics and Evaluation (IHME). GBD Compare Data Visualization. Seattle, WA: IHME, University of Washington, 2016. Available online from: http://vizhub.healthdata.org/gbd-compare. Accessed 15 Jan 2017.

5. Ministry of Health, Malawi. National Action Plan for Prevention and Management of Non-Communicable Diseases in Malawi (2012-2016). 2013

6. K.P. Msyamboza, B. Ngwira, T. Dzowela, et al. The burden of selected chronic non-communicable diseases and their risk factors in Malawi: nationwide STEPS survey. PLoS One. 2011;6(5):e20316.

7. Ministry of Health, Malawi. Malawi Health Sector Strategic Plan: 2011-2016. 2011. Available online from: http://www. nationalplanningcycles.org/sites/default/files/country_docs/Malawi/2_ malawi_hssp_2011_-2016_final_document_1.pdf. Accessed 5 Jun 2017.

8. World Health Organisation (WHO). Package of Essential Noncommunicable (PEN) Disease Interventions for Primary Health Care in Low-Resource Settings. 2010. Available online from: http:// www.who.int $/ \mathrm{nmh} /$ publications/essential_ncd_interventions_lr settings.pdf?ua=1. Accessed 25 Jan 2017.

9. World Diabetes Foundation (WDF). WDF14-938: Diabetes and Hypertension Control in Malawi. WDF grant. 2015. Available online from: https://www.worlddiabetesfoundation.org/projects/malawiwdf14-938. Accessed 15 Jan 2017.

10. Oxford Poverty and Human Development Initiative. Global Multidimensional Poverty Index Databank. 2016. OPHI, University of Oxford.

11. Institute for Health Metrics and Evaluation (IHME). About GBD. Seattle, WA: IHME, University of Washington, 2016. Available at: http://www.healthdata.org/gbd/about. Accessed 8 June 2017.

12. The Lancet NCDI Poverty Commission. Scientific Protocol for Lancet Commission on Reframing NCDs and Injuries for the Poorest Billion (NCDI Poverty Commission) - National Level Analyses. 2016. 